\title{
Ghost Imaging Lidar via Sparsity Constraints in Real Atmosphere
}

\author{
Mingliang Chen, Enrong Li, Wenlin Gong, Zunwang Bo, Xuyang Xu, Chengqiang Zhao, \\ Xia Shen, Wendong Xu, Shensheng Han \\ Key Laboratory for Quantum Optics, Center for Cold Atom Physics of CAS, Shanghai Institute of Optics and Fine Mechanics, \\ Chinese Academy of Sciences Shanghai, China \\ Email: sshan@mail.shcnc.ac.cn, cml2008@mail.siom.ac.cn
}

Received 2013

\begin{abstract}
We present a series of results acquired at a 2-kilometer distance using our lidar system under several weather conditions, clear, cloudy, light rain, moderately foggy, and night. The experimental results show that ghost imaging lidar via spar-sity constraints can realize imaging in all these weather conditions.
\end{abstract}

Keywords: Ghost Imaging; Lidar; Atmosphere; Reconstruction Algorithm

\section{Introduction}

In recent years, thanks to the development of laser technology and laser detection techniques, several lidar techniques have been developed, such as spot scanning imaging lidar [1], 3D imaging lidar [2-4] and synthetic aperture imaging lidar [5-7]. Ghost imaging lidar via sparsity constraints (GISC lidar) is a newly developed imaging lidar technique based on the ghost imaging principle [8]. It gets target global information with a highly sensitive bucket detector, and the image is reconstructed with some algorithms. At present, most researches about ghost imaging in atmosphere are theoretical $[9,10]$ or laboratory experiments [11, 12]. We shall present the GISC lidar experimental results under several weather conditions.

\section{Experiments}

Our experimental setup is shown in Figure 1. Pseudothermal light is produced by a $532 \mathrm{~nm}$ laser pulse passing through a rotating ground glass. The light is split into two paths by a beam splitter, of which the light field intensity of reference and test beam accounts respectively for $95 \%$ and $5 \%$ of the total energy of the incident light. In our GISC lidar system, the source plane optical field is imaged respectively to the reference plane and the target plane through the lenses $\mathrm{L}_{r}$ and $\mathrm{L}_{t}[8,13]$. The target reflects the test beam light back to the receiving system. The aperture of the receiving telescope is $0.42 \mathrm{~m}$ with 5.0 $\mathrm{m}$ focal length. The photons collected by the receiving system pass through an interference filter with $1 \mathrm{~nm}$ half bandwidth, then received by a photomultiplier tube (PMT). Reference beam light passes through lens $\mathrm{L}_{r}$ and im- ages on a CCD detector. The intensity distribution of light field $I(m \times n)$ is reshaped into a row vector $I(1 \times N)$ [14, 15], in which $N=m \times n$. After $M$ observations, we obtain measurement matrix $A(M \times N)$ of $M$ row vectors $I(1 \times N)$, and $Y(M \times 1)$ from the $M$ signals recorded by the PMT. In our experiments, $M=3000, m=n=200$. The outdoor detection distance is $2 \mathrm{~km}$. The scene and target are shown in Figure 2. The target with a size of $54 \mathrm{~cm} * 58 \mathrm{~cm}$ is a two-dimensional resolution panel, with two groups of slits (threeslit, and double-slit), whose center-to-center distances between slits is $8 \mathrm{~cm}, 12 \mathrm{~cm}$, respectively.

Ghost imaging reconstruction algorithm plays an essential role in GISC lidar system. Although the linear reconstruction algorithm was first developed [16], nonlinear algorithms are usually used in a GISC lidar system since they better exploits the information from the collected data $[8,14,15]$. We developed a nonlinear

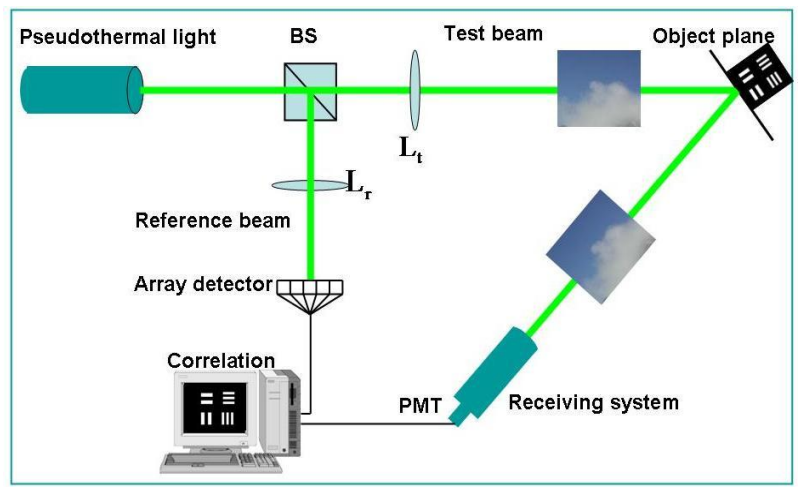

Figure 1. Experimental setup of GIL system. 


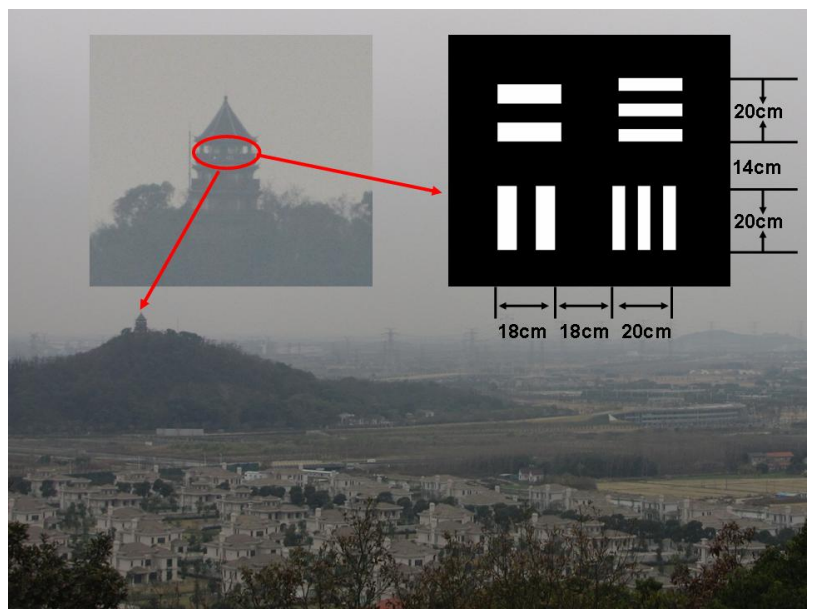

Figure 2. Scene and target of $\mathbf{2} \mathbf{k m}$ field experiments.

reconstruction algorithm based on TV constraint and Krush-Kuhn-Tucker conditions [17], solving the following optimization program:

$$
\begin{aligned}
& \max -\|Y-A X\|_{2}^{2} / 2-\tau\|X\|_{T V} \\
& \text { s.t. }-x_{i} \leq 0,
\end{aligned}
$$

where $\|X\|_{T V}$ is the total variation of the image $X$, $x_{i}$ the i-th element of $X$, and $\tau$ a constant.

The experimental scene is clearly visible in both clear and cloudy conditions, as shown in the Figure 3(a1), (b1). Under the condition of light rain, as shown in Figure $3(\mathbf{c 1})$, the outline of the $2 \mathrm{~km}$ distance mountain, where the target is situated, is still dimly visible. Whereas in a moderate fog as shown in Figure 3(d1), the aforesaid mountain is invisible. Though traditional imaging has no problem imaging the target in clear as well as cloudy weather conditions, GISC lidar gets images of a much higher signal-to-noise ratio (SNR), as shown in Figure 3(a), (b). In light rain and moderate fog, affected by low visibility caused by rain drops and fog, traditional imaging cannot see the target, as shown in Figure 3(c), (d). By active illumination, spatial filtering, and highly sensitive signal detection, GISC lidar manages to extract the target's weak signals despite the low visibility and high noises. The results of night experiments are shown in Figure 3(e). Apparently, traditional imaging with equal irradiation energy laser illumination is unable to get the target's image, whereas GISC lidar can. Columns Figure 3(3), 3(4) compare the images reconstructed by GI linear reconstruction algorithm and TV nonlinear reconstruction algorithm using the same experimental data. Again, apparently, the quality of the images reconstructed by the TV algorithm is better than those reconstructed by the GI algorithm.

\section{Conclusions}

In conclusion, GISC lidar's ability of imaging in real (a)

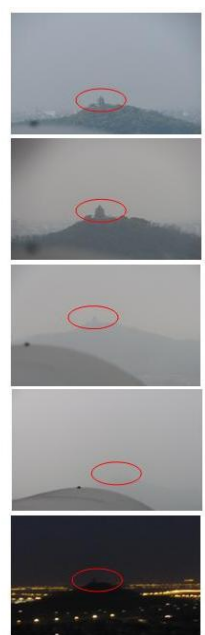

(1)

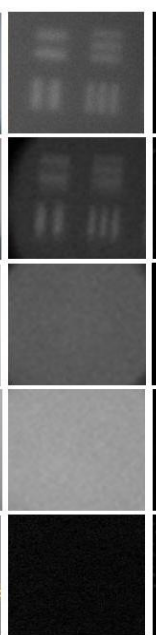

(2)

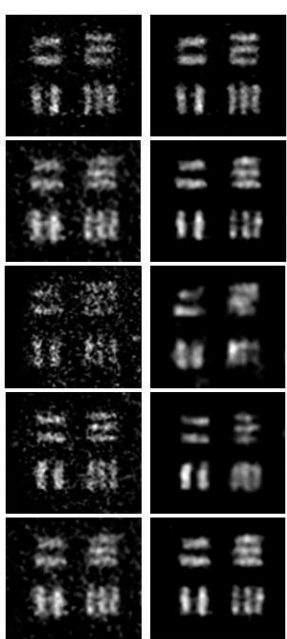

(3)
(4)
Figure 3. GIL experimental results. Weather and atmosphere conditions: (a) clear, (b) cloudy, (c) light rain, (d) moderately foggy, (e) night. (1) Scenes of Field Experimental, (2) the results of traditional imaging, (3) images reconstructed by GI, (4) images reconstructed by TV.

atmosphere is demonstrated. It features in its quality imaging in rainy, misty and foggy, and night conditions. It is a new type of imaging lidar that can work in some weather conditions where traditional imaging methods can't work. The unique superiority of GISC lidar will point to the development of its potential application value in various areas.

\section{Acknowledgements}

Our thanks go to all the colleagues co-worked with us for the outdoor experiments. This research is supported by the Hi-Tech Research and Development Program of China under Grant Project No.2011AA120101 and 2011AA120102.

\section{REFERENCES}

[1] S. M. Spuler and S. D. Mayor, "Scanning Eye-Safe Elastic Backscatter Lidar at 1.54um," Journal of Atmospheric and Oceanic Technology, Vol. 22, No. 6, pp. 696-703, 2005.doi:10.1175/JTECH1755.1

[2] R. M. Marino, T. Stephens, R. E. Hatch, J. L. McLaughlin, J. G. Mooney and O'Brien, et al. “A Compact 3 D Imaging Laser Radar System Using Geiger-Mode APD Arrays-System and Measurements," Proceedings of SPIE, Vol. 5086, No. 1-15, 2003. doi:10.1117/12.501581

[3] Gleckler, A. D. and A. Gelbart. "Multiple-slit streak tube imaging lidar (MS-STIL) applications,” Proceedings SPIE, Vol. 4035, No. 266-278, 2000. doi:10.1117/12.397800

[4] N. D. Hardy and J. H. Shapiro, "Computational Ghost Imaging Versus Imaging Laser Radar for 3D Imaging," arXiv preprint arXiv:1212.3253, 2012. unpublished. 
[5] S. M. Beck, J. R. Buck, W. F. Buell, R.P. Dickinson, D. A. Kozlowski, N. J. Marechal, et al. "Synthetic-aperture imaging laser radar: laboratory demonstration and signal processing,” Applied Optics, Vol. 44, No. 35, pp. 7621-7629, 2005. doi:10.1364/AO.44.007621

[6] M. Bashkansky, R. L. Lucke, E. Funk, L. J. Rickard and J. Reintjes, “Two-Dimensional Synthetic Aperture Imaging in the Optical Domain,” Optics letters, Vol. 27, No. 22, 2002, pp. 1983-1985. doi:10.1364/OL.27.001983

[7] L. Liren, Z. Yu and Z. Yanan, "A Large-Aperture SynThetic Aperture Imaging Ladar Demonstrator and Its Verification in Laboratory Space," Acta Optica Sinica, Vol. 31, No. 9, 2011, p. 0900112. doi:10.3788/AOS201131.0900112

[8] C. Zhao, W. Gong, M. Chen, E. Li, H. Wang, W. Xu, et al., "Ghost Imaging Lidar Via Sparsity Constraints," Applied Physics Letters, Vol. 101, 2012, p. 141123. doi:10.1063/1.4757874

[9] J. Cheng, "Ghost imaging through turbulent atmosphere," Optics express, Vol. 17, No.10, 2009, pp.7916-7921. doi:10.1364/OE.17.007916

[10] P. Zhang, W. Gong, X. Shen and S. Han, "Correlated imaging through atmospheric turbulence,” Physical Review A, Vol. 82, 2010, p. 033817. doi:10.1103/PhysRevA.82.033817

[11] R. E. Meyers, K. S. Deacon, Y. Shih, "Positive-negative turbulence-free ghost imaging,” Applied Physics Letters, Vol.100,No.13,2012,p.131114. doi:10.1063/1.3698158

[12] R. E. Meyers, , K. S. Deacon, A. D. Tunick and Y. Shih, "Virtual Ghost Imaging through Turbulence and Obscurants Using Bessel Beam Illumination," Applied Physics Letters,Vol.100,No.6, 2012,pp.061126-061129. doi:10.1063/1.3684613

[13] P. Zhang, W. Gong, X. Shen, D. Huang and S. Han, "Improving resolution by the second-order correlation of light fields," Optics letters,Vol.34, No.8, 2009,pp. 1222-1224. doi:10.1364/OL.34.001222

[14] O. Katz, Y. Bromberg and Y. Silberberg, "Compressive ghost imaging," Applied Physics Letters,Vol.95, No.13, 2009,pp.131110-131112. doi:10.1063/1.3238296

[15] W. Gong and S. Han, "Super-Resolution Ghost Imaging via Compressive Sampling Reconstruction,” arXiv preprint arXiv:0910.4823, 2009.

[16] W. Gong and S. Han, "A Method to Improve the Visibility of Ghost Images Obtained by Thermal Light,” Physics Letters A, Vol. 374, No.8, 2010, pp. 1005-1008. doi:10.1016/j.physleta.2009.12.030

[17] E. Li, M. Chen, W. Gong, H. Wang and S. Han, “A Fast Converging Sparse Reconstruction Algorithm in Ghost Imaging," Journal of Electronics, No.6,2012, pp. 617-620. doi:10.1007/s11767-012-0878-8 